\title{
Public health measures, radiotherapy, and the novel coronavirus outbreak
}

\author{
Antonio Cassio Assis Pellizzon, MD, MsC, PhD \\ Radiation Oncology, A.C. Camargo Cancer Center, Sao Paulo, Brazil
}

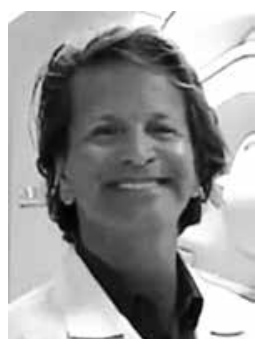

Address for correspondence: Antonio Cassio Assis Pellizzon, Head of Radiation Oncology Department, A.C. Camargo Cancer Center, Rua Prof Antonio Prudente 211, Liberdade, Sao Paulo, 01509-020 Brazil, phone: +5511 21895000, ext. 2265, 2267, 2271, e-mail: acapellizzon@accamargo.org.br

In health practice, the quarantine refers to the separation of persons or communities who are exposed to an infectious disease. Isolation, in contrast, applies to the "separation" of persons who are known to be infected. Of importance is that isolation and quarantine can be voluntary or imposed by law. However, these classical public health measures are usually of limited utility for highly transmissible diseases. Other tools that the public health has at hand are social distancing and community containment. The primary goal of such measures is to prevent person-to-person spread of infection in order to interrupt the transmission of disease.

In this view, oncologic patients need to be evaluated and balanced whether to continue, stop, or postpone the start of treatments. For radiotherapy with curative intent, prolongation of the prescribed overall treatment time has been linked to inferior clinical outcomes $[1,2,3]$. Prospective and retrospective studies have also shown that treatment interruptions and prolongation can increase the risk of local recurrence by up to $2 \%$ per day for certain malignancies [4].

Despite the current coronavirus pandemic, radiation departments already suffer from non-compliance of patients. The published non-compliance rate is significantly associated with higher age, male gender, advanced stage of tumor, administration of concomitant chemotherapy, and greater travel distance of patients to avail radiotherapy [5].

The understanding of radiobiology and recent advances of technologies had evolved to modified fractionations, which can shorten overall treatment times. Motivations for developing protracted radiotherapy regimens relate not only to tumor control, but also to benefit the patients and health services in terms of convenience and cost. Besides brachytherapy, other altered fractions treatments such as moderated hypofractionation (IGRT) or ablative treatment regimens using stereotactic body radiotherapy (SBRT), are measures, which can reduce departments workload, but all of these are given in a certain number of fractions for each tumor site, in general ranging from 3 to 20 fractions.

During quarantine or social isolation, it is relevant to protect and support oncologic patients, particularly those under radiotherapy whose treatments shall not be interrupted. The use of modified fractions and actions to maintain patients in radiation treatment should be individually tailored. In a recent publication, just 24 days after Wuhan lockdown, Wei et al. investigated situations of radiation therapy centers in five provinces in China (Guangdong, Hunan, Anhui, Zhejiang, and Jiangxi). In these areas, there are 160 linear accelerators in 74 hospitals. Based on survey results from all hospitals, $88 \%$ of them declared to provide radiation treatment for patients with negative screening results. For patients asymptomatic with an epidemic travel history, $70 \%$ of hospitals believed that these patients could be treated after 14 days of isolation. For confirmed coronavirus-infected patients who had been cured, all hospitals showed a more cautious attitude, with up to $39 \%$ of hospitals indicating that they would not provide radiation treatment for such patients. In terms of protective measures, $93 \%$ of radiotherapy centers have created restricted areas for patients receiving radiation treatment. For the safety of cancer patients and medical staff, the strategy to manage patients who require radiation included: 1 . All patients were screened before the initial evaluation for radiation therapy and classified into confirmed cases, suspected cases, cases that required medical isolation, and cases with negative screening results. For patients with negative screening, if they were early-stage cancer patients or if radiation therapy intervention was urgently needed, the nearest radiotherapy center was considered; for patients with locally advanced tumors, a neoadjuvant chemotherapy regimen, followed by an appropriate delay of radiation therapy was recommended. For patients who were already receiving radiotherapy, treatment should be continued, and radiotherapy doses appropriately adjusted based on biological effects. 2. Radiation treatment room and nearby areas should be thoroughly disinfected during treatment intervals. 3 . Reasonable protective measures should be provided to patients and radiotherapy staff. 4. Patients were encouraged to use online platforms for medical consultation, and to receive treatment in the nearest hospital to reduce an exposure to crowds [6].

The present pandemic places cancer patients in an even worse situation. It is particularly important to determine patients' risk of disease progression and coronavirus exposure. Decisions based on the risk of cancer progression need to be undertaken for each individual patient. Interventions such as communication with other support health professionals can reduce distress in patients receiving radiotherapy during the present coronavirus pandemic. 


\section{References}

1. Thames HD, Kuban D, Levy LB et al. The role of overall treatment time in the outcome of radiotherapy of prostate cancer: an analysis of biochemical failure in 4839 men treated between 1987 and 1995. Radiol Oncol 2010; 96: 6-12.

2. Perez CA, Grigsby PW, Castro-Vita H, Lockett MA. Carcinoma of the uterine cervix. I. Impact of prolongation of overall treatment time and timing of brachytherapy on outcome of radiation therapy. Int J Radiat Oncol Biol Phys 1995; 32: 1275-1288.

3. Fowler JF, Lindstrom MJ. Loss of local control with prolongation in radiotherapy. Int J Radiat Oncol Biol Phys 1992; 23: 457-467.

4. Bese NS, Hendry J, Jeremic B. Effects of prolongation of overall treatment time due to unplanned interruptions during radiotherapy of different tumor sites and practical methods for compensation. Int J Radiat Oncol Biol Phys 2007; 68: 654-661.

5. Gupta S, Rastogi K, Bhatnagar AR et al. Compliance to radiotherapy: A tertiary care center experience. Indian J Cancer 2018; 55: 166-169.

6. Wei $\mathrm{W}$, Jiang $\mathrm{H}$, Chen $\mathrm{W}$ et al. How should we implement radiotherapy for cancer patients in China during the endemic period of COVID-19? Radiother Oncol 2020; 147: 100-102. 